
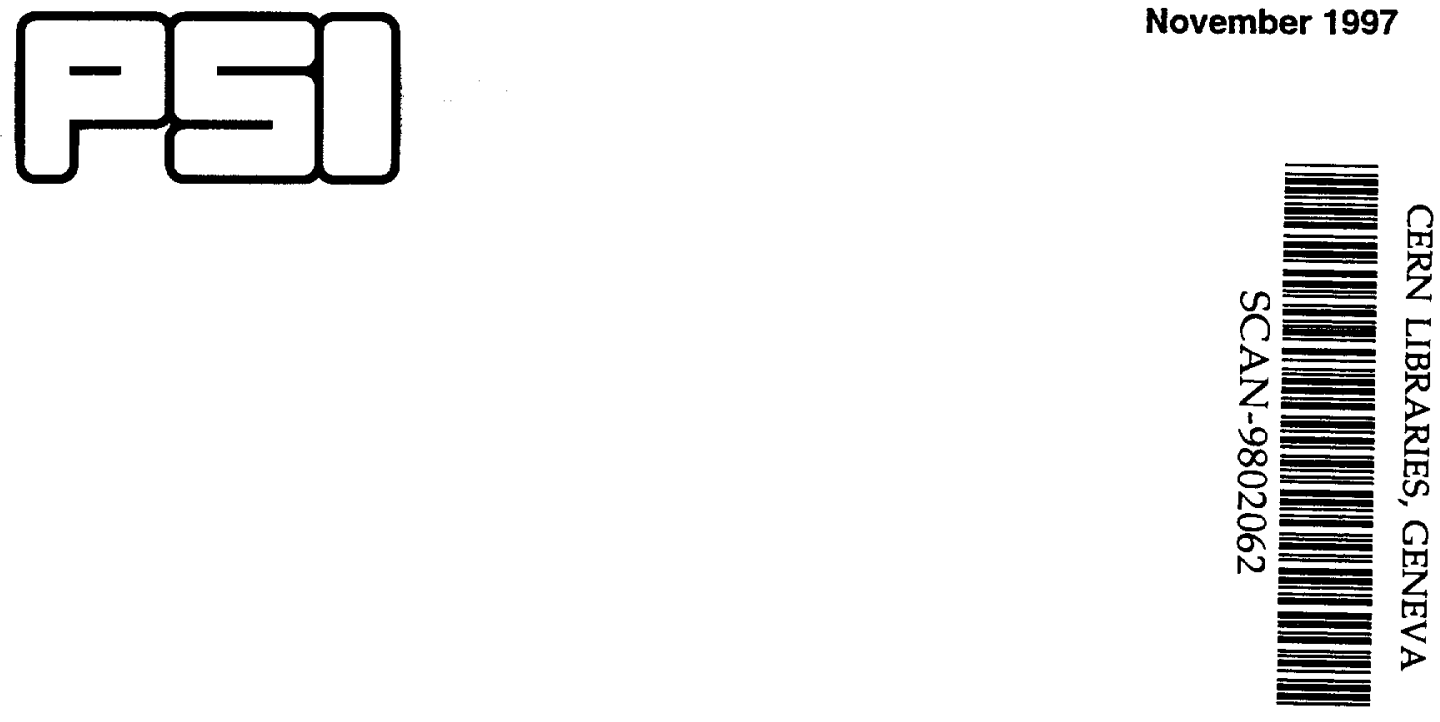

Sw9803

\title{
A Consistent Calculation of Dispersion Corrections in Elastic Electron-Deuteron Scattering
}

T. Herrmann 1,2 and R. Rosenfelder ${ }^{2}$

${ }^{1}$ Fakultät f. Physik, Universität Kaiserslautern, D-67663 Kaiserslautern, Germany

2Paul Scherrer Institut, $\mathrm{CH}-5232$ Villigen PSI, Switzerland 


\title{
A Consistent Calculation of Dispersion Corrections
}

\author{
in \\ Elastic Electron-Deuteron Scattering
}

\author{
T. Herrmann ${ }^{1,2, *}$ and R. Rosenfelder ${ }^{2}, \dagger$ \\ ${ }^{1}$ Fakultät f. Physik, Universität Kaiserslautern, D-67663 Kaiserslautern, Germany \\ 2 Paul Scherrer Institut, CH-5232 Villigen PSI, Switzerland
}

\begin{abstract}
We calculate the contribution of virtual second-order excitations of the deuteron by integrating numerically over a generalized inelastic structure function $S\left(\mathbf{q}_{1}, \mathbf{q}_{2}, \omega\right)$. This structure function, as well as the ground state density, are evaluated analytically using the separable Yamaguchi Swave $N N$-potential which gives a fair description of low-energy deuteron properties and nuclear polarization shifts. In the static case excellent numerical agreement is found by comparing the second-order Born results with a partial-wave calculation. In the non-static case recoil corrections are also taken into account but only Coulomb excitations, which should be dominant for small momentum transfers, are retained. In contrast to previous calculations the present approach avoids uncontrolled approximations like the closure approximation or mixing of different models for ground and excited states. We show that the closure approximation with a fixed average excitation energy is unable to reproduce our numerical results which are found to be smaller than in previous estimates, negative and dependent both on scattering angle and incident electron energy. An analysis of experimental scattering cross sections at low momentum transfer is performed including static Coulomb and dispersion corrections. In agreement with a recent analysis it is found that the Coulomb corrections increase the charge radius by $0.012 \mathrm{fm}$ whereas our dispersion corrections lead to a decrease of only $0.003 \mathrm{fm}$. This gives a deuteron radius of $(1.968 \pm 0.006) \mathrm{fm}$ and a charge radius of $(2.130 \pm 0.010) \mathrm{fm}$.
\end{abstract}

"present address: Institute of Molecular Biology and Biophysics, ETHZ, CH-8093 Zürich, Switzerland

${ }^{\dagger}$ corresponding author 


\section{Introduction}

There has been considerable interest recently in the precise value of the deuteron radius $[1-7]$. This is, of course, due to the fact that the deuteron is the fundamental two-body system in nuclear physics and its size therefore related to the $N N$-interaction, but also due to dramatic improvements in laser spectroscopy of hydrogen and deuterium atoms which are now sensitive even to subtle nuclear effects $[8,9]$. Whereas the dominant finite-size effect gives the desired information about the deuteron radius, virtual excitations of the weakly bound deuteron (called nuclear polarization) also have to be considered since they contribute about $19 \mathrm{kHz}$ to the deuteron-proton isotope shift in the $1 S-2 S$ transition. This has to be compared with an experimental uncertainty which has shrunk from 20 $\mathrm{kHz}$ in 1993 [8] to about $2 \mathrm{kHz}$ in 1996 [10] with further reductions in progress. Several theoretical calculations of the nuclear polarization shift in deuterium [11 - 15] now exist which - when using realistic $N N$-potentials - agree within $2 \%$. Efforts are also under way to measure the deuteron radius in muonic transitions [16] where the finite size effect is much larger. Realistic nuclear polarization shifts in this system have been evaluated in Ref. [13].

In contrast, the classical method to measure nuclear radii by elastic electron scattering experiments nearly always has neglected virtual excitations of the target, here called dispersion corrections. The main reason for ignoring them has been that no reliable calculation is available but there is also the general belief that they are "small". However, Coulomb corrections, i.e. the distortion of electron waves by the static potential are also "small" for a $Z=1$-nucleus like the deuteron but a recent re-analysis of the available scattering data [17] has shown that they shift the deuteron charge radius deduced from electron scattering to a value nearly compatible with that from laser spectroscopy. The question, how important dispersion corrections are, is thus a quantitative one and in view of the on-going quest for a precise deuteron radius it is certainly worthwhile to re-examine them in elastic electron-deuteron scattering. This will be done in the present work.

A reliable calculation of dispersion corrections is much more demanding than the evaluation of nuclear polarization shifts. In the latter case only one number is required, whereas in the former case an additional scattering amplitude, which interferes with the dominant static amplitude, has to be calculated. This interference depends on scattering angle and incident electron energy, and therefore it is absolutely essential that the same nuclear model be used both for the static and the non-static amplitude. In addition, for nuclear polarization in light nuclei, the bound lepton can be considered as quasi-static, transferring practically zero momentum to the nucleus, whereas for dispersion corrections the electron is ultra-relativistic and there is an appreciable net momentum transfer. As we will see, this requires knowledge of a generalized structure function of two momentum variables whereas for nuclear polarization in light nuclei one has to deal with the usual inelastic structure function of one momentum variable which is much simpler. Even in this case, however, one needs information about all excited states of the nucleus which, in general, is not available. Therefore, further drastic approximations (like the closure approximation) are usually applied which make the outcome unreliable and often inconsistent. This is particularly disastrous for the interference with the static amplitude and explains the twisted history of calculations of dispersions corrections. Fortunately the deuteron is one of the rare cases where these structure functions can be calculated and we will not employ any of these approximations. Given the complexity of the numerical calculation we restrict ourselves in the following to simple, separable $N N$-interactions which allow for an analytic evaluation of the generalized structure function. Also we consider only longitudinal excitations which should 


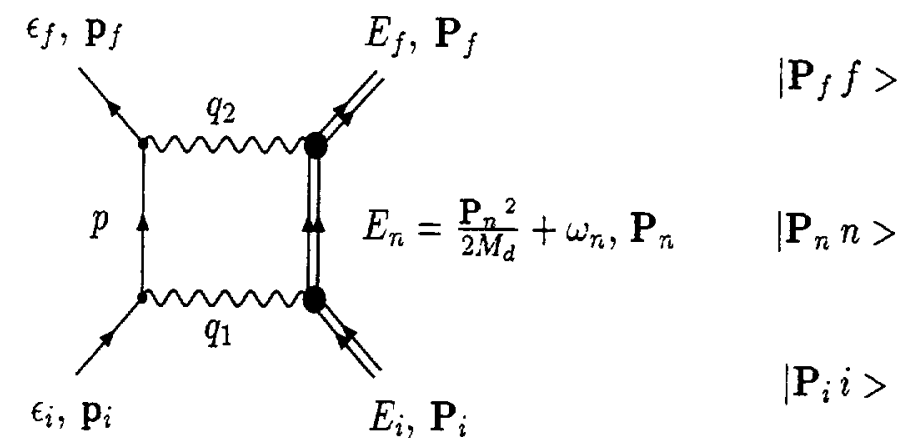

Figure 1: Direct two-photon exchange graph for dispersion corrections and kinematical notations.

be dominant for small scattering angles. Under these kinematical conditions the main non-static contributions are low-energy virtual excitations into the continuum for which the separable S-wave potentials should give an adequate description. It is also in the small-angle region that the radius of the deuteron is mainly determined.

\section{Two-photon exchange}

The theory of dispersion corrections is well developed and we will follow the classic paper by Friar and Rosen [18]. The direct graph for two-photon exchange is shown in Fig. 1.

Together with the crossed two-photon exchange diagram it leads to the following $S$-matrix

$$
S_{f i}^{(2)}=\left(4 \pi e^{2}\right)^{2} \sqrt{\frac{m_{e}^{2}}{\epsilon_{i} \epsilon_{f} V^{2}}}(2 \pi)^{4} \delta^{4}\left(q+P_{i}-P_{f}\right) \int \frac{d^{4} p}{(2 \pi)^{4}} t_{\mu \nu} D^{\mu \rho}\left(q_{2}\right) D^{\nu \tau}\left(q_{1}\right) T_{\rho \tau}
$$

where $e^{2}=1 / 137.036$ is the fine-structure constant, $D^{\mu \rho}$ the photon propagator and $t_{\mu \nu}$ and $T_{\rho \tau}$ are the electron and nuclear Compton amplitudes respectively ${ }^{a}$. The latter contains the nuclear currents and the "sea-gull" term which we neglect in the following (in nuclear polarization calculations it only gives a very small contribution [12]). We use the following kinematical variables

$$
\begin{aligned}
& q \equiv p_{i}-p_{f}=P_{f}-P_{i}=q_{1}+q_{2} \\
& q_{1}=p_{i}-p, \quad q_{2}=p-p_{f}
\end{aligned}
$$

and we will work in the Coulomb gauge. Our main approximation is to consider only the longitudinal part of the interaction. This is the leading term in a $1 / M$-expansion ( $M$ being the nucleon mass) of the nuclear currents

$$
\left\langle\mathbf{P}_{f}, f\left|J_{\mu}(0)\right| \mathbf{P}_{i}, i>=\delta_{\mu 0}\left\langle f|\rho(\mathbf{q})| i>+\mathcal{O}\left(\frac{1}{M}\right) \simeq \delta_{\mu 0} G_{p}(\mathbf{q})<f\left|\sum_{j} \exp \left(i \mathbf{q} \cdot \mathbf{r}_{j}^{\prime}\right)\right| i>.\right.\right.
$$

Here $\mathbf{r}_{j}^{\prime}$ is the co-ordinate of $j$-th proton relative to the center-of-mass ( $=\mathbf{r} / 2$ in the deuteron with $\mathbf{r}$ being the distance between proton and neutron) and $G_{p}(\mathbf{q})$ the proton form factor (we neglect the neutron form factor). Since convection and spin currents have additional factors $|\mathbf{q}| / M$ compared to

\footnotetext{
${ }^{\circ}$ The Bjorken-Drell conventions are used throughout this work.
} 
the charge operator this should be a valid approximation for low momentum transfers. When only $T_{00}$ is retained in Eq. (1) the second-order $S$-matrix can be written as

$$
S_{f i}^{(2)}=i\left(4 \pi e^{2}\right)^{2} \sqrt{\frac{m_{e}^{2}}{\epsilon_{i} \epsilon_{f} V^{2}}}(2 \pi)^{4} \delta^{4}\left(q+P_{i}-P_{f}\right) \bar{u}_{f}\left(p_{f}\right) \gamma_{0} u_{i}\left(p_{i}\right) \cdot \mathcal{T}_{L}
$$

with

$$
\begin{aligned}
\mathcal{T}_{L}= & \int \frac{d^{3} p}{(2 \pi)^{3}} \int d \omega \frac{1}{\mathbf{q}_{1}^{2} \mathbf{q}_{2}^{2}} S_{L}\left(\mathbf{q}_{1}, \mathbf{q}_{2}, \omega\right)\left\{\left[k+\mathbf{p} \cdot\left(A^{(+)} \mathbf{s}+A^{(-)} \mathbf{q}\right)\right] \frac{1}{\mathbf{p}^{2}-k^{2}-i 0}\right. \\
& \left.+\left[1-\frac{\mathbf{p}}{|\mathbf{p}|} \cdot\left(A^{(+)} \mathbf{s}+A^{(-)} \mathbf{q}\right)\right] \frac{\tilde{k}-k}{2(k+|\mathbf{p}|-i 0)(|\mathbf{p}|+\tilde{k}-i 0)}\right\} .
\end{aligned}
$$

Here

$$
\begin{aligned}
A^{( \pm)} & =\frac{1}{\mathbf{q}^{2} \mathbf{s}^{2}-(\mathbf{q} \cdot \mathbf{s})^{2}}\left[\mathbf{q}^{2}\left(\epsilon_{i} \pm \epsilon_{f}\right)-\mathbf{q} \cdot \mathbf{s}\left(\epsilon_{i} \mp \epsilon_{f}\right)\right], \quad \mathbf{s}=\mathbf{p}_{i}+\mathbf{p}_{f} \\
k(\omega, \mathbf{p}) & =\epsilon_{i}-\omega+\frac{\mathbf{P}_{i}{ }^{2}-\left(\mathbf{P}_{i}+\mathbf{p}_{i}-\mathbf{p}\right)^{2}}{2 M_{d}} \\
\tilde{k}(\omega, \mathbf{p}) & =\epsilon_{f}+\omega+\frac{\left(\mathbf{P}_{i}+\mathbf{p}-\mathbf{p}_{f}\right)^{2}-\mathbf{P}_{i}{ }^{2}}{2 M_{d}} .
\end{aligned}
$$

Note that $\omega$ is the internal excitation energy of the deuteron, the recoil effects being contained in $k, \tilde{k}$ and the energy-momentum conserving $\delta$-function. The complete nuclear information is encoded in the generalized longitudinal structure function

$$
S_{L}\left(\mathbf{q}_{1}, \mathbf{q}_{2}, \omega\right)=F_{0}\left(\mathbf{q}_{1}\right) F_{0}\left(\mathbf{q}_{2}\right) \delta(\omega)+\underbrace{\left.\sum_{n \neq 0}^{\infty} \delta\left(\omega+\omega_{0}-\omega_{n}\right)<0\left|\rho\left(\mathbf{q}_{2}\right)\right| n><n\left|\rho\left(\mathbf{q}_{1}\right)\right| 0\right\rangle}_{=: S_{L}^{\text {inelastic }}} .
$$

As usual its elastic part is fully determined by the elastic form factor $F_{0}(\mathbf{q})$. Friar and Rosen [18] have neglected the second term in the curly bracket of Eq. (5) since it is suppressed approximately by a factor $\omega / \epsilon_{i}$. In our calculation we easily can keep this term, although it will turn to make only a small contribution.

We now can work out the cross section up to order $e^{6}$ from the interference of Eq. (4) with the first-order $S$-matrix

$$
S_{f i}^{(1)}=i Z\left(4 \pi e^{2}\right) \sqrt{\frac{m_{e}^{2}}{\epsilon_{i} \epsilon_{f} V^{2}}}(2 \pi)^{4} \delta^{(4)}\left(q+P_{i}-P_{f}\right) \bar{u}_{f}\left(p_{f}\right) \gamma^{0} u_{i}\left(p_{i}\right) \frac{F_{0}(\mathbf{q})}{\mathbf{q}^{2}}
$$

of a spinless deuteron. This is most conveniently done in the laboratory frame since the convection current of the whole nucleus is then contained in $T_{00}$ [18]. Including the proton ("dipole") form factor $G_{p}(\mathbf{q})=\left(1+\mathbf{q}^{2} /\left(0.71 \mathrm{GeV}^{2}\right)\right)^{-2}$ and neglecting the electron mass one obtains [19]

$$
\left(\frac{d \sigma}{d \Omega}\right)_{\text {lab }}^{(1+2)}=\sigma_{\text {Mott }} f_{\text {recoil }}\left[F_{0}^{2}(\mathbf{q}) G_{\mathrm{p}}^{2}(\mathbf{q})+\frac{8 \pi e^{2}}{Z} F_{0}\left(\mathbf{q}^{2}\right) G_{\mathbf{p}}(\mathbf{q}) \mathbf{q}^{2} \operatorname{Re}\left(\mathcal{T}_{L}\right)\right] \text {. }
$$

Here

$$
\sigma_{\mathrm{Mott}}=\frac{\left(Z e^{2}\right)^{2}}{4 \epsilon_{i}^{2} \sin ^{4}(\Theta / 2)} \cos ^{2} \frac{\Theta}{2}
$$


is the Mott cross section and

$$
f_{\text {recoil }}=\left[1+2 \frac{\epsilon_{i}}{M_{d}} \sin ^{2} \frac{\Theta}{2}\right]^{-1}
$$

the usual recoil factor ( $\Theta$ denotes the laboratory scattering angle and $M_{d}$ the deuteron mass ${ }^{b}$ ). Note that the interference term involves the real part of $\mathcal{T}_{L}$, i.e the principal value of the propagators.

It is worthwhile to discuss some special cases of Eq. (9) : for potential scattering one should set $M_{d}=\infty, G_{p}=1$ and retain only the elastic part of the generalized structure function. The second line in Eq. (5) then vanishes and one obtains the expression given by Lewis [21]. The result from the second-order Born approximation can then be compared with that from a partial-wave calculation in which the Dirac equation is solved numerically for each partial wave; this serves as a check on the accuracy of the numerical integration. In Fig. 2 the relative difference

$$
\bar{\Delta}_{\text {coul }}=\left[\left(\frac{d \sigma}{d \Omega}\right)_{\text {part }}-\left(\frac{d \sigma}{d \Omega}\right)_{\text {Born }}\right] /\left(\frac{d \sigma}{d \Omega}\right)_{\text {part }}
$$

is plotted.

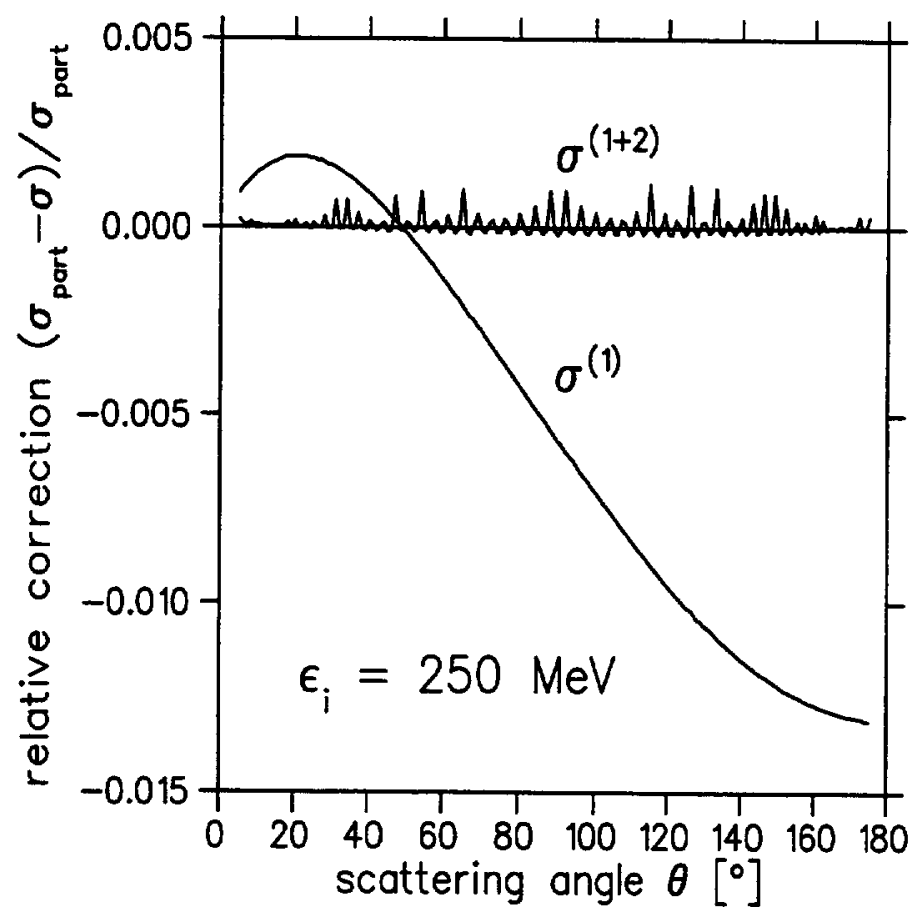

Figure 2: Relative difference between the exact partial-wave cross section and the first and second order Born approximation for an electron energy of $250 \mathrm{MeV} .2 \times 72$ Gaussian points were used for the numerical integration in each dimension. The charge distribution is the one from the Yamaguchi $N N$-potential (see Appendix, Eq. (A4)) with $\beta=286 \mathrm{MeV}$ and no proton form factor.

\footnotetext{
${ }^{b}$ In kinematical quantities, like recoil factor and momentum transfer, we employ the exact value $M_{d}=1875.613 \mathrm{MeV}$. In dynamical quantities we usually substitute $M_{d}=2 M$ with $M=938.9 \mathrm{MeV}$ being the nucleon mass.
} 
As can be seen, the second-order or "Coulomb corrections" raise the cross section at small scattering angles from the first-order Born result and then change sign. It is also seen that the agreement of the second-order Born calculation with the partial-wave cross section is very good and below the per-mil level, with the remaining wiggles due to the numerical integration of the 3 -dimensional momentum integral. In principle, one integration (say over an angle) could have been performed analytically but we have chosen not to do so since any additional momentum dependence from form factors, recoil terms etc. can be included easily in our program. The outcome is highly non-trivial since one has to treat carefully the usual infra-red singularities of the Coulomb potential and to evaluate numerically a principal value integral. This was done following the method of Ref. [20]. Similar investigations have been done long ago with emphasis on particular charge distributions or high-energy approximations $[21,22]$. Of course, for a point nucleus, $\mathcal{T}_{L}$ can be calculated analytically and one obtains the well-known correction [23]

$$
\frac{d \sigma^{(2)}}{d \Omega}=\sigma_{\mathrm{Mott}} \cdot Z e^{2} \pi \frac{\sin (\Theta / 2)}{1+\sin (\Theta / 2)}
$$

to the Mott cross section.

\section{Dispersion corrections}

Turning now to the dispersion corrections for elastic electron-deuteron scattering we need the generalized structure function $S_{L}\left(\mathbf{q}_{1}, \mathbf{q}_{2}, \omega\right)$. In the present work we employ the simple separable Yamaguchipotential [24]

$$
V\left(\mathbf{p}, \mathbf{p}^{\prime}\right)=-\frac{\lambda}{M} \frac{1}{\mathbf{p}^{2}+\beta^{2}} \frac{1}{\mathbf{p}^{\prime 2}+\beta^{2}}
$$

to generate both the elastic form factor and the generalized structure function in a consistent and analytic form. For convenience and further reference we give these expressions in the Appendix. We have checked them by evaluating the sum rule

$$
\int_{\omega_{0}}^{\infty} d \omega S_{L}^{\text {inelastic }}\left(\mathbf{q}_{1}, \mathbf{q}_{2}, \omega\right)=F_{0}\left(\mathbf{q}_{1}-\mathbf{q}_{2}\right)-F_{0}\left(\mathbf{q}_{1}\right) F_{0}\left(\mathbf{q}_{2}\right)
$$

numerically from $\omega_{0}=2.2245 \mathrm{MeV}$ to $\omega_{\max }=1000 \mathrm{MeV}$ for different vector values of $\mathbf{q}_{1}, \mathbf{q}_{2}$. Excellent agreement to within $10^{-5}$ for Eq. (15) was obtained.

Allowing for virtual excitations of the deuteron, a 4-dimensional integral for the quantity $\mathcal{T}_{L}$ in Eq. (5) now has to be evaluated numerically. Again this was done by Gauss-Legendre integration, usually with 24 points per dimension. Typical run times were 2 hours per angle on a SUN SPARC station. We also have checked the numerical accuracy at selected angles by using up to $2 \times 32$ Gaussian points [19]. Figure 3 shows the relative correction

$$
\bar{\Delta}_{\text {disp }}=\left[\left(\frac{d \sigma}{d \Omega}\right)_{n \geq 0}-\left(\frac{d \sigma}{d \Omega}\right)_{n=0}\right] /\left(\frac{d \sigma}{d \Omega}\right)_{n=0}
$$

to the static cross section obtained at incident electron energies of 125,250 and $500 \mathrm{MeV}$. Proton form factor and recoil corrections are included in the calculation. 
With the strength fixed to reproduce the deuteron binding energy $\omega_{0}$, there is only one free parameter $\beta$ left in the Yamaguchi potential (14). While the original work [24] fixed it at a value of $\beta=286 \mathrm{MeV}$ from the experimental triplet scattering length, a fit to the deuteron matter radius would require values between 255 and $259 \mathrm{MeV}$ (see also Fig. 7). Here the higher value is associated with the rms-radius deduced by Sprung and Wu [4] and the lower value with the one from Martorell et al. [14]. However, from calculations of nuclear polarization shifts in electronic and muonic atoms one knows that (at least for very small momentum transfers) the electric dipole polarizability $\alpha_{E}$ of the deuteron plays the decisive role. Indeed, the difference between the calculation in Ref. [12] which also used the Yamaguchi potential and the one in Ref. [13] which employed realistic potentials was mainly caused by too small a value of the polarizability from Yamaguchi's original $\beta$-parameter. Adopting the value $\alpha_{E}=0.6328(17) \mathrm{fm}^{3}$ [25] and using the analytical formula (A8) for this quantity, requires

$$
\beta=268 \mathrm{MeV} .
$$

This value has been adopted in the present calculation, with results plotted in Fig. 3.

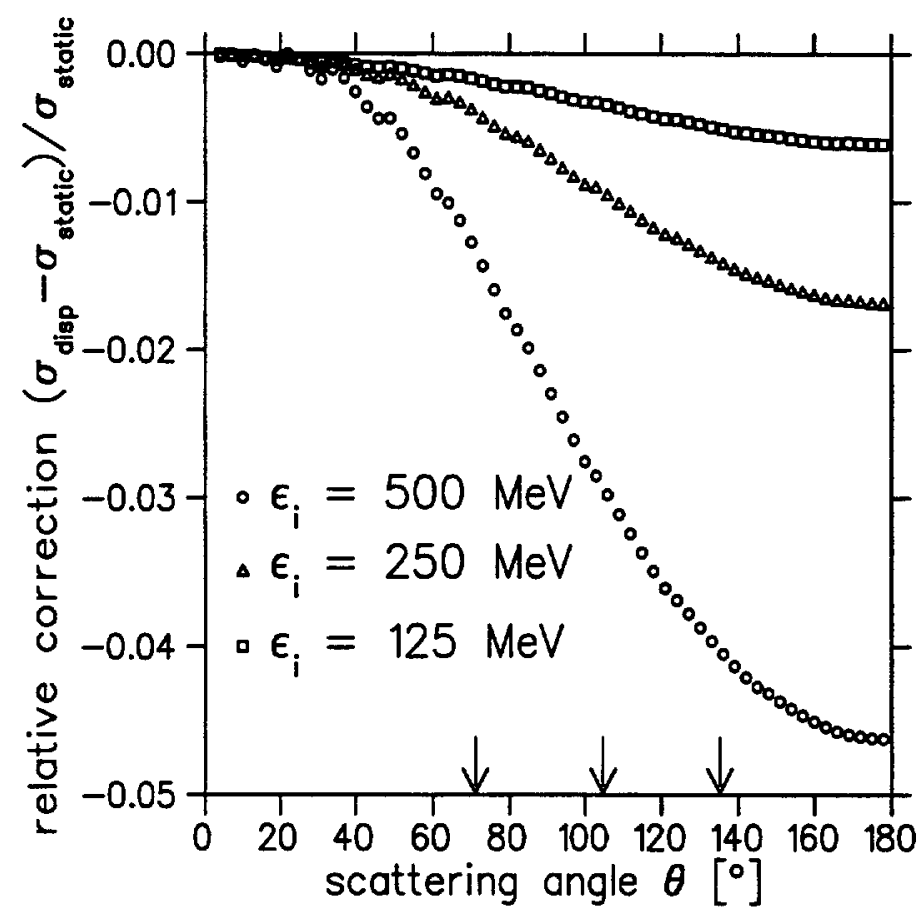

Figure 3: Relative dispersion corrections for elastic electron-deuteron scattering.

Although this value of $\beta$ leads to a matter radius of $1.946 \mathrm{fm}$ (which is too low) we do not believe that the relative correction is very much affected by the inability of the Yamaguchi potential to describe both observables precisely. This is because the elastic electron-deuteron cross section is smooth and non-diffractive, so that the relative correction to the static cross section should be predicted with better accuracy than the cross section itself. Of course, a calculation with realistic $N N$-potentials would avoid these shortcomings but that seems unfeasible and unnecessary at the moment. But it would be more important to include transverse excitations which should have some influence at 
larger scattering angles. Conversely this implies that the dispersion corrections calculated using only Coulomb excitations cannot be considered as reliable for too large scattering angles. Including transverse contributions the elastic cross section for unpolarized electron-deuteron scattering in first order Born approximation is given by the well-known formula

$$
\frac{d \sigma}{d \Omega}^{(1)}=\sigma_{\text {Mott }} f_{\text {recoil }} \cdot\left\{F_{L}^{2}+F_{T}^{2}\left[\frac{1}{2}+\tan ^{2}\left(\frac{\Theta}{2}\right)\right]\right\} \text {. }
$$

In our simplified S-wave description of the deuteron the transverse elastic form factor is due to the spin current only and reads $[27]$

$$
F_{T}^{2}(\mathbf{q})=\frac{\mathbf{q}^{2}}{3 M^{2}} \mu_{d} G_{p}^{2}(\mathbf{q}) F_{0}^{2}(\mathbf{q})
$$

where $\mu_{d}=\mu_{p}+\mu_{n}$ is the magnetic moment of the deuteron. Therefore its contribution relative to the charge scattering is

$$
\Delta_{\text {mag }}=\frac{\mathbf{q}^{2}}{3 M^{2}} \mu_{d}^{2}\left[\frac{1}{2}+\tan ^{2}\left(\frac{\Theta}{2}\right)\right] .
$$

Since we have neglected the transverse form factor we may take as a rough (and rather arbitrary) criterion for neglecting transverse excitations that the transverse elastic contribution should be less than $10 \%$ of the Coulombic part. The corresponding scattering angles are marked by an arrow in Fig. $3^{c}$.

\section{Discussion}

It is interesting to compare the dispersion corrections to the static 2-photon exchange corrections, because Ref. [17] found the Coulomb corrections to be important in their analysis of measured $e-d$ cross sections. This is shown in Fig. 4 for an incident electron energy of $500 \mathrm{MeV}$. It can be seen that the dispersion corrections are more important than static Coulomb corrections for scattering angles larger than about 60 degrees. However, in view of the neglected transverse excitations, it is uncertain whether this would hold for a complete calculation.

As Fig. 3 shows a strong dependence of the calculated dispersion corrections on electron energy and scattering angle, it is worthwhile to present them also as a function of energy and squared momentum transfer. This is done in Fig. 5 and reveals an approximate linear $\mathbf{q}^{2}$-dependence for both low and high momentum transfers, albeit with different slopes. In addition, we observe that for smaller electron energy (at fixed momentum transfer), larger dispersion corrections are obtained than for higher energy.

In order to understand these features qualitatively we investigate Eq. (5) in the limit $M_{d} \rightarrow \infty$ where $A^{(+)}=2 \epsilon_{i} / \mathbf{s}^{2}, A^{(-)}=0$ and neglect the second term in the curly bracket of Eq. (5). Furthermore we concentrate on small angle scattering $\Theta \rightarrow 0^{\circ}$. With $\mathbf{q}_{1}=\mathbf{p}_{i}-\mathbf{p}=-\mathbf{q}_{2}$ we then obtain

$$
\mathcal{T}_{L}^{\text {inelastic }}(\Theta=0) \simeq \int \frac{d^{3} p}{(2 \pi)^{3}} \int d \omega \frac{S_{L}^{\text {inelastic }}\left(\mathbf{q}_{1}, \mathbf{q}_{2}, \omega\right)}{\left(\mathbf{p}-\mathbf{p}_{i}\right)^{4}} \frac{\epsilon_{i}-\omega+\mathbf{p} \cdot \mathbf{p}_{i} / \epsilon_{i}}{\mathbf{p}^{2}-\left(\epsilon_{i}-\omega\right)^{2}-i 0}
$$

\footnotetext{
${ }^{c}$ This does not necessarily imply that transverse excitations are totally negligible below these scattering angles because the transverse inelastic structure function at large momentum transfer is proportional to the incoherent sum $\mu_{p}^{2}+\mu_{n}^{2} \simeq 11.46$ of magnetic moments whereas the elastic part is proportional to the coherent sum $\left(\mu_{p}+\mu_{n}\right)^{2} \simeq 0.77$.
} 


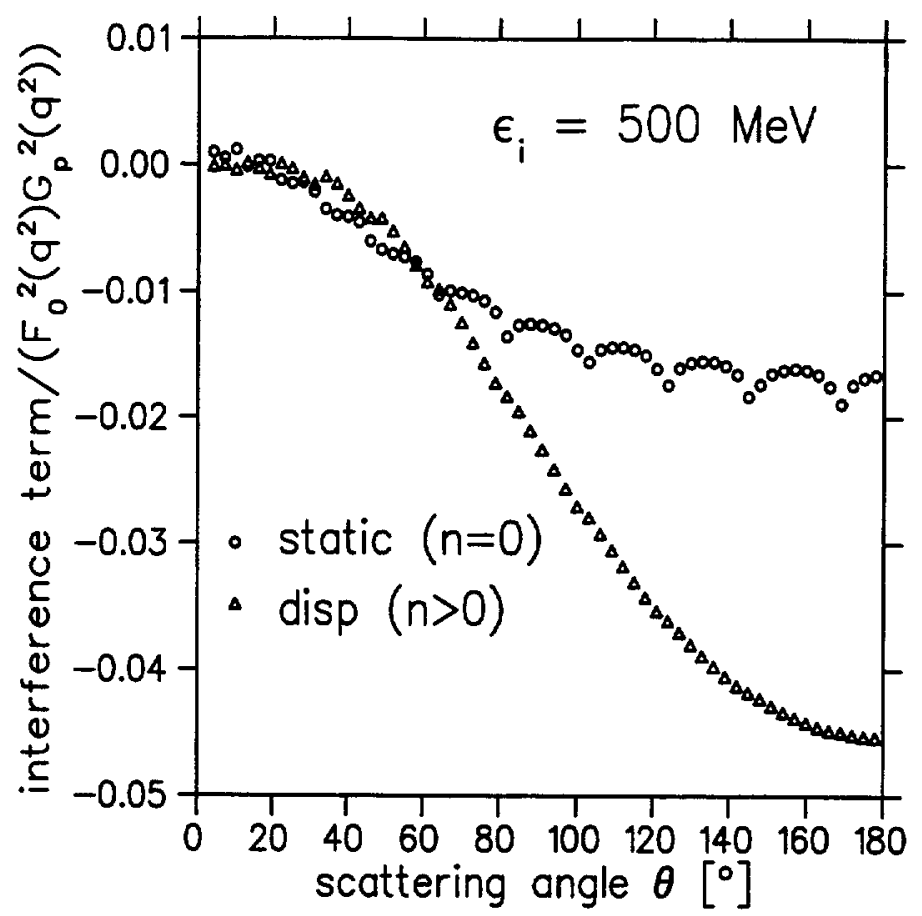

Figure 4: Static and non-static two-photon corrections for an incident electron energy of $500 \mathrm{MeV}$.

Let us assume that all relevant excitation energies $\omega$ are small compared to the incident electron energy $\epsilon_{i}$ (this is borne out below by evaluating the mean average excitation energy). After neglecting $\omega$ in the last factor of Eq. (21) we can perform the $\omega$-integration by means of the sum rule (15) and obtain

$$
\mathcal{T}_{L}^{\text {inelastic }}(\Theta=0) \simeq \int \frac{d^{3} p}{(2 \pi)^{3}} \frac{F_{0}\left(2\left(\mathbf{p}-\mathbf{p}_{i}\right)\right)-F_{0}^{2}\left(\mathbf{p}-\mathbf{p}_{i}\right)}{\left(\mathbf{p}-\mathbf{p}_{i}\right)^{4}} \frac{\epsilon_{i}+\mathbf{p} \cdot \mathbf{p}_{i} / \epsilon_{i}}{\mathbf{p}^{2}-\epsilon_{i}^{2}-i 0}
$$

This is, of course, the closure approximation with zero mean excitation energy. Writing $\mathbf{k}=\mathbf{p}-\mathbf{p}_{i}$, the principal value integral for the real part of $\mathcal{T}_{L}$ can be performed with the result

$$
\operatorname{Re} \mathcal{T}_{L}^{\text {inelastic }}(\Theta=0) \simeq \frac{1}{4 \pi^{2}} \int_{0}^{\infty} d k \frac{F_{0}(2 k)-F_{0}^{2}(k)}{k^{3}} H\left(\frac{k}{2 \epsilon_{i}}\right)
$$

where

$$
H(x)=2 x+\left(1-x^{2}\right) \ln \left|\frac{1+x}{1-x}\right| .
$$

Note that $H(x) \geq 0$, grows for small arguments like $4 x$, reaches a maximum at $x=0.8336$ and decreases for large $x$ like $4 /(3 x)$. The limit $x \rightarrow 0$ is more relevant since the deuteron form factor restricts the momentum $k$ to be of the order of its inverse radius $1 / R$ and for the energies of interest $\epsilon_{i} \cdot R \gtrsim 1$. If we adopt this further approximation we obtain

$$
\operatorname{Re} \mathcal{T}_{L}^{\text {inelastic }}(\Theta=0) \simeq \frac{1}{\epsilon_{i}} \frac{1}{2 \pi^{2}} \int_{0}^{\infty} d k \frac{1}{k^{2}}\left[F_{0}(2 k)-F_{0}^{2}(k)\right]=: \frac{C_{0}}{\epsilon_{i}}
$$

(where $C_{0}$ is a constant) or for the relative change with respect to the first-order Born approximation

$$
\Delta_{\text {disp }}^{\text {closure }}(\Theta \rightarrow 0) \simeq 8 \pi e^{2} C_{0} \frac{q^{2}}{\epsilon_{i}} .
$$




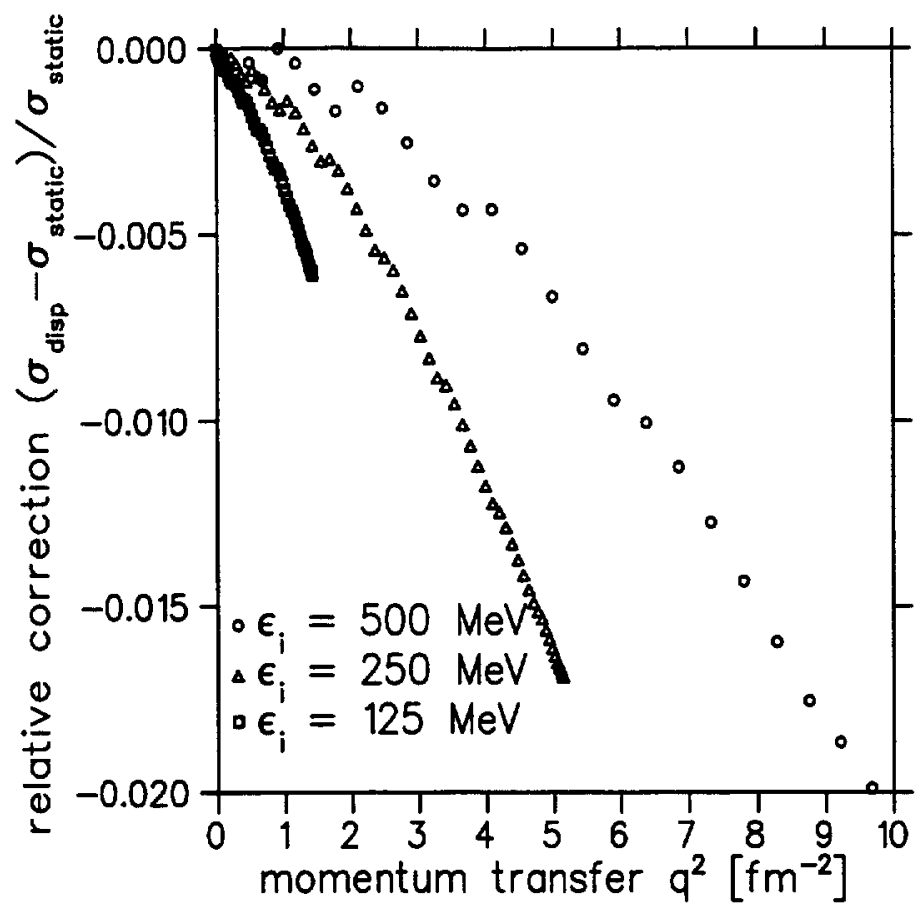

Figure 5: Relative dispersion corrections as a function of the squared momentum transfer $\mathbf{q}^{2}$ for different electron energies.

Thus the closure approximation with zero average excitation energy explains the linear $\mathbf{q}^{2}$-dependence at small scattering angles and the dependence on the incident electron energy observed in the numerical results. It also predicts the negative sign of the dispersion corrections: the constant $C_{0}$ is negative because the combination of elastic form factors

$$
F_{0}(2 k)-F_{0}^{2}(k) \stackrel{k \rightarrow 0}{\longrightarrow}-\frac{k^{2}}{3}\left\langle r^{2}\right\rangle+\ldots
$$

is negative for small $k$ which contribute most due to the weighting factor $1 / k^{2}$ in the integral in Eq. (25). Indeed, a numerical evaluation with the Yamaguchi form factor $(\beta=268 \mathrm{MeV})$ gives $C_{0}=-1.60 \cdot 10^{-4} \mathrm{MeV}^{-1}$ which leads to the simple estimate

$$
\Delta_{\text {disp }}^{\text {closure }}(\Theta \rightarrow 0) \simeq-1.14 \frac{\mathrm{q}^{2}\left[\text { in fm }^{-2}\right]}{\epsilon_{i}[\text { in MeV }]}
$$

Unfortunately this is only in semi-qualitative agreement with our exact numerical evaluation of the dispersion corrections as, for example, given in the $8^{\text {th }}$ column of Table $1:$ Eq. (27) overestimates the corrections by a factor $1.5-2.5$ for the smallest scattering angles.

At this point it is interesting to compare with previous calculations found in the literature. There are only a few theoretical investigations of dispersion corrections which dealt explicitly with the deuteron $[28,20]$. Among these the work of Bottino and Ciochetti [20] is the most extensive. These authors use the dipole approximation and a parametrization of the photo-absorption cross section for the deuteron to derive nearly energy-independent dispersion corrections and a change of less than $1 \%$ in the rms-radius. Roughly their approach corresponds to a (questionable) substitution of Eq. (27) 
into Eq. (23):

$$
\operatorname{Re} \mathcal{T}_{L}^{\text {inelastic, dipole }}(\Theta=0) \simeq-\frac{\left\langle r^{2}\right\rangle}{12 \pi^{2}} \int_{0}^{\infty} d x \frac{1}{x} H(x)=-\frac{\left\langle r^{2}\right\rangle}{24}
$$

which, indeed, is independent of the electron energy. It leads to a relative correction

$$
\Delta_{\text {disp }}^{\text {dipole }}(\Theta \rightarrow 0) \simeq-\frac{\pi e^{2}}{3} \mathbf{q}^{2}\left\langle r^{2}\right\rangle \simeq-0.035 \mathrm{q}^{2}\left[\mathrm{in} \mathrm{fm}^{-2}\right]
$$

which is much too large in magnitude (for example at $\epsilon_{i}=80.1 \mathrm{MeV}, \Theta=30^{\circ}$ it overestimates the numerical result by more than a factor of 4 ). Since the estimate (30) only depends on the momentum transfer it would lead - if taken seriously - to a relative change in the rms-radius of $-\pi e^{2} / 2 \simeq-1.1 \%$ which, however, is unrealistic. The dipole (or long wavelength) approximation amounts to approximating the generalized inelastic structure function by

$$
S_{L}^{\text {inelastic, dipole }}\left(\mathbf{q}_{1}, \mathbf{q}_{2}, \omega\right) \simeq \mathbf{q}_{1} \cdot \mathbf{q}_{2} \frac{\sigma_{\gamma}(\omega)}{4 \pi^{2} e^{2} \omega}
$$

where $\sigma_{\gamma}(\omega)$ is the photo-absorption cross section which fulfills the sum rule $\int_{0}^{\infty} d \omega \sigma_{\gamma}(\omega) / \omega=$ $4 \pi^{2} e^{2}\left\langle r^{2}\right\rangle / 3$. This approximation is only valid for very small incident electron energies for which $\epsilon_{i}\left\langle r^{2}\right\rangle^{1 / 2} \ll 1$. In addition, in ref. [20] the sign of the correction to the rms-radius seems to be wrong: these authors claim that dispersion corrections increase the radius which obviously contradicts our results ${ }^{d}$.

Krall and Salpeter, on the other hand, in a very early paper [28] with rather crude approximations obtain (mostly) negative dispersion corrections and estimate that the charge radius of the deuteron decreases by $-0.010 \mathrm{fm}$ which is in at least qualitative agreement with our findings. However, it should be emphasized again that all these approximations are either invalid or unrealistic for analyzing measured electron-deuteron cross sections. We conclude that at present there is no short-cut and no substitute for the "extremely tedious" [15] numerical integration over the loop variables.

Finally it is worthwhile to check whether the closure approximation with a non-zero average excitation energy would work. For this purpose we have determined for each scattering angle and for two incident electron energies the average excitation energy $\bar{\omega}$ in the curly bracket of Eq. (5) which would produce the same results as the exact calculation. The results are shown in Fig. 6 and clearly show that $\bar{\omega}$ would have to be a complicated function both of scattering angle and electron energy. Thus no evaluation of dispersion corrections with a fixed average excitation energy correctly predicts the change in the cross section. Note that in very forward direction $\bar{\omega}$ is close to the deuteron binding energy which is the least energy to be transferred in order to excite the target. It is also reassuring that for small scattering angles these average excitation energies stay at the order of a few $\mathrm{MeV}$ so that $k R \ll 1$ and the use of a potential which only acts in the relative $\mathrm{S}$-wave is justified a posteriori.

\footnotetext{
${ }^{d}$ We see no way how an overall sign error could have occured in our calculation: As a simple check one can try to estimate the elastic part of $\mathcal{T}_{L}(\theta \rightarrow 0)$ in the same way as the inelastic part. Then one has to insert $F_{0}^{2}>0$ instead of the form factor combination into Eq. (23). Since $H(x)$ is positive this gives a positive Coulomb correction at small scattering angles in full agreement with Fig. 2 and numerous numerical and analytical investigations. In the inelastic case $F_{0}(2 k)-F_{0}^{2}(k)$ is dominantly negative as argued above. Negative dispersion corrections lead to a smaller true radius since the same experimental cross section which contains these effects is (wrongly) reproduced by a larger radius in a static analysis.
} 


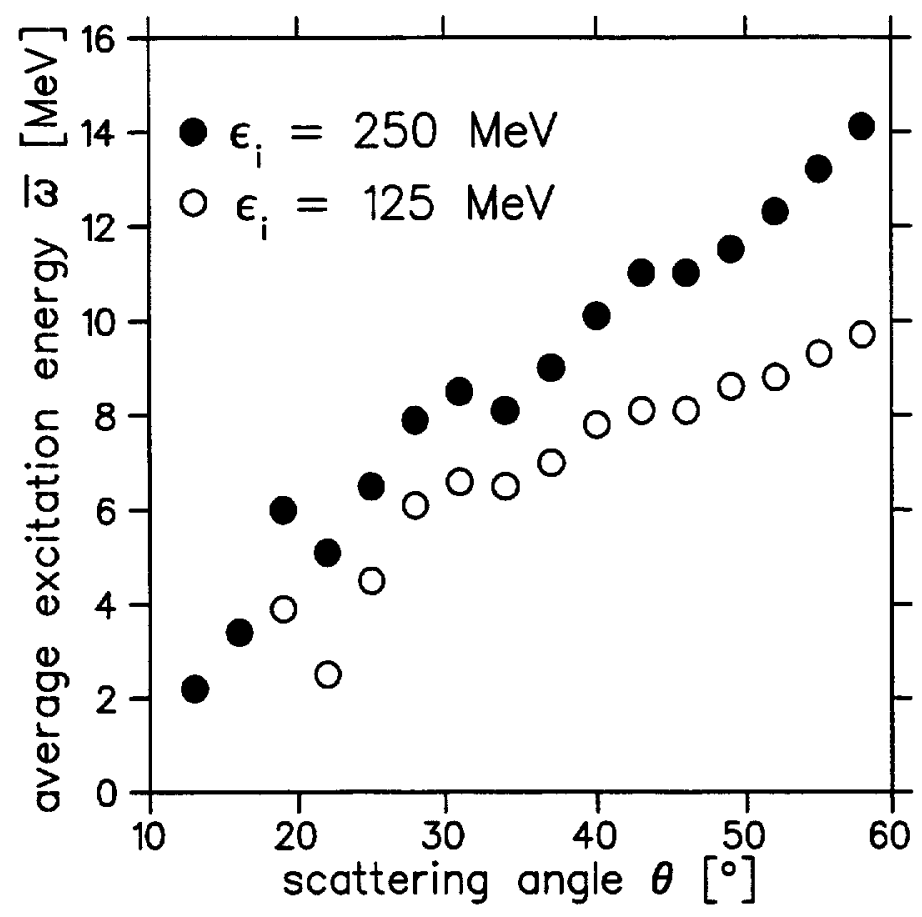

Figure 6: Average excitation energy $\bar{\omega}$ for which the exact dispersion correction are reproduced in closure approximation. Fluctuations are due to the numerical integration.

\section{Analysis of experimental scattering data with dispersion correc- tions}

In order to assess the importance of dispersion corrections for the determination of the deuteron radius we now will analyze experimental electron scattering data at low momentum transfer. Whereas a rough description of the electron-deuteron interaction was sufficient to describe the (small) dispersion corrections we now have to take into account other effects as well. We will write the $e-d$ cross section as (see Eq. (9))

$$
\frac{d \sigma}{d \Omega}=\sigma_{\text {Mott }} f_{\text {recoil }} F_{0}^{2}(\mathbf{q}) G_{p}^{2}(\mathbf{q})[1+\Delta]
$$

and subsume all contributions and corrections which go beyond the first order Born approximation for a spinless target into the quantity $\Delta$. (Note that $\bar{\Delta}_{i}=\Delta_{i} /\left(1+\Delta_{\text {coul }}\right) \simeq \Delta_{i} ; i=$ coul, disp have been plotted in Figs. 2,3 .) We are interested in the body (or matter or point nucleon) form factor which has the low-q expansion

$$
F_{0}(\mathbf{q}) \equiv\left\langle 0\left|e^{i \mathbf{q} \cdot \mathbf{r} / 2}\right| 0\right\rangle \stackrel{\mathbf{q}^{2} \rightarrow 0}{\longrightarrow} 1-\frac{1}{6} \mathbf{q}^{2} r_{m}^{2}+\ldots
$$

Meson-exchange and other two-body corrections add a small correction $\delta r_{m}$ so that we are actually determining the radius $r_{E d}$ defined by

$$
r_{E d}^{2}=r_{m}^{2}+\delta r_{m}^{2}
$$


If we substitute Eq. (33) into Eq. (32) we see that the corrections induce a change in the radius

$$
\delta r_{E d}^{2}=\lim _{\mathbf{q} \rightarrow 0} \Delta \cdot \frac{6}{\mathbf{q}^{2}} \frac{d \sigma / d \Omega}{\sigma_{\text {Mott }} f_{\text {recoil }} G_{p}^{2}}
$$

which has the same sign as the low-q limit of these corrections, i.e. as pointed out before, negative dispersion corrections reduce the radius from its static value.

We will now discuss in more detail the additional corrections which have to be considered for a realistic analysis of the data. We first recall that the longitudinal part of the $e-d$ cross section is given by $[29,30,31]$

$$
F_{L}^{2}=G_{E d}^{2}\left(Q^{2}\right)+\frac{Q^{4}}{18} G_{Q d}^{2}\left(Q^{2}\right)
$$

where $Q^{2}=-q^{2}=\mathbf{q}^{2}-q_{0}^{2} \simeq \mathbf{q}^{2}$ and $G_{E d}(0)=1$ (charge), $G_{Q d}(0)=Q_{d}$ (quadrupole moment) ${ }^{e}$. The charge form factor $G_{E d}\left(Q^{2}\right)$ is made up of the body form factor $F_{0}$, the proton and neutron form factors and the so-called Darwin-Foldy term:

$$
G_{E_{d}}=\langle 0|\rho(\mathbf{q})| 0\rangle=\left(1-\frac{\mathbf{q}^{2}}{8 M^{2}}\right)\left[G_{E p}\left(Q^{2}\right)+G_{E_{n}}\left(Q^{2}\right)\right] F_{0}(\mathbf{q}) .
$$

This relation can be derived from the well-known non-relativistic reduction of the electromagnetic current operator (see, e.g. ref. [32], Appendix D)

$$
\hat{\rho}(\mathbf{q})=\sum_{i=p, n}\left\{F_{1}^{(i)}-\left[\frac{\mathbf{q}^{2}}{8 M^{2}}-\frac{i}{4 M^{2}} \mathbf{q} \cdot\left(\boldsymbol{\sigma}_{i} \times \mathbf{p}_{i}\right)\right]\left(F_{1}^{(i)}+2 F_{2}^{(i)}\right)\right\} e^{i \mathbf{q} \cdot \mathbf{r}_{i}^{\prime}}+\mathcal{O}\left(\frac{1}{M^{3}}\right)
$$

which replaces Eq. (3). Here $F_{1 / 2}$ are the Dirac/Pauli form factors and $\mathbf{r}_{i}^{\prime}$ the relative co-ordinates of proton and neutron in the deuteron. It is customary to use the Sachs form factors

$$
\begin{aligned}
& G_{E}^{(i)}\left(Q^{2}\right)=F_{1}^{(i)}\left(Q^{2}\right)-\frac{Q^{2}}{4 M^{2}} F_{2}^{(i)}\left(Q^{2}\right) \\
& G_{M}^{(i)}\left(Q^{2}\right)=F_{1}^{(i)}\left(Q^{2}\right)+F_{2}^{(i)}\left(Q^{2}\right)
\end{aligned}
$$

which (in the Breit frame) have a better interpretation as Fourier transforms of charge and magnetization distributions. If one neglects the spin-dependent term in Eq. (38) (it vanishes for an S-wave deuteron) one obtains Eq. (37) up to order $1 / M^{2}$.

We have analyzed the electron scattering data of Simon et al. [33] including static Coulomb and dispersion corrections ${ }^{f}$. We have included all $15 e-d$ cross sections with $Q^{2}<0.4 \mathrm{fm}^{-2}$ which were measured relative to the $e-p$ cross sections [35]. In this way the systematic and normalization errors are much smaller than for an absolute measurement: we have taken the statistical errors given in Table 2 of ref. [33] and have added linearly the normalization error of $0.13 \%$ for the ratio (see caption of Table 1 in this reference).

We then proceeded in the following steps:

\footnotetext{
${ }^{e}$ In our simplified S-wave deuteron, $G_{Q d}\left(Q^{2}\right) \equiv 0$, of course.

${ }^{f}$ The data of Berard et al. [34] could not be used since the published cross sections are only given as function of $Q^{2}$.
} 


\begin{tabular}{|c|r|c|c|c|c|c|c|c|}
\hline$\epsilon_{i}[\mathrm{MeV}]$ & $\Theta\left[^{\circ}\right]$ & $Q^{2}\left[\mathrm{fm}^{-2}\right]$ & $\sigma_{\text {rel }}$ & $\Delta_{\text {mag }}$ & $\Delta_{\text {quad }}$ & $\Delta_{\text {coul }}$ & $\Delta_{\text {disp }}$ & $R(\Delta R)$ \\
\hline \multirow{3}{*}{80.1} & 30 & 0.044 & 0.9362 & 0.0003 & 0.0000 & 0.0040 & -0.0003 & $0.9709(14)$ \\
& 70 & 0.212 & 0.7461 & 0.0023 & 0.0002 & 0.0049 & -0.0010 & $0.8836(19)$ \\
& 75 & 0.237 & 0.7197 & 0.0028 & 0.0003 & 0.0048 & -0.0012 & $0.8704(19)$ \\
& 80 & 0.263 & 0.6993 & 0.0034 & 0.0003 & 0.0047 & -0.0013 & $0.8605(19)$ \\
& 85 & 0.290 & 0.6781 & 0.0042 & 0.0004 & 0.0046 & -0.0013 & $0.8498(21)$ \\
& 90 & 0.316 & 0.6543 & 0.0051 & 0.0005 & 0.0044 & -0.0014 & $0.8371(22)$ \\
& 100 & 0.368 & 0.6121 & 0.0077 & 0.0006 & 0.0041 & -0.0017 & $0.8139(20)$ \\
& 40 & 0.263 & 0.7021 & 0.0018 & 0.0003 & 0.0027 & -0.0008 & $0.8636(23)$ \\
& 45 & 0.328 & 0.6417 & 0.0024 & 0.0005 & 0.0024 & -0.0009 & $0.8320(27)$ \\
& 50 & 0.398 & 0.5928 & 0.0031 & 0.0007 & 0.0021 & -0.0010 & $0.8061(24)$ \\
& 40 & 0.265 & 0.6984 & 0.0018 & 0.0003 & 0.0026 & -0.0008 & $0.8615(81)$ \\
& 45 & 0.330 & 0.6412 & 0.0024 & 0.0005 & 0.0024 & -0.0010 & $0.8318(32)$ \\
& 28 & 0.236 & 0.7172 & 0.0014 & 0.0003 & 0.0021 & -0.0006 & $0.8704(19)$ \\
& 30 & 0.270 & 0.6910 & 0.0017 & 0.0003 & 0.0020 & -0.0007 & $0.8578(24)$ \\
& 35.49 & 0.363 & 0.6254 & 0.0024 & 0.0006 & 0.0017 & -0.0005 & $0.8247(33)$ \\
& 35 & & & & & & & \\
\hline
\end{tabular}

Table 1: Incident energies, scattering angles, four momentum transfers and measured (relative) cross sections from Simon, Schmitt and Walther [33]. The various corrections $\Delta_{i}$ are explained in the text. The ratio $R$ is defined in Eq. (43) and used to extract the deuteron radius.

1. We have taken out the Mott cross section and the recoil factor to define

$$
\sigma_{\text {rel }}=\frac{d \sigma}{d \Omega} /\left(\sigma_{\text {Mott }} f_{\text {recoil }}\right)
$$

which is listed in the $4^{\text {th }}$ column of Table 1 .

2. We have corrected for magnetic and quadrupole contributions by using Eq. (20) with the experimental value $\mu_{d}=0.85741$ and

$$
\Delta_{\text {quad }}=\frac{\mathbf{q}^{4}}{18} Q_{d}^{2}
$$

with $Q_{d}=0.2859 \mathrm{fm}^{2}$. The value of these corrections are given in the $5^{\text {th }}$ and $6^{\text {th }}$ column of Table 1, respectively and seen to be small or even tiny in the case of quadrupole scattering. Static Coulomb corrections have been calculated either in the second Born approximation (as a by-product of the dispersion corrections) or by a partial-wave code. In the latter case the Yamaguchi density (A4) with $\beta=254 \mathrm{MeV}$ was folded with the proton and neutron form factors (parametrizations from refs. $[35,36]$ ) plus the Darwin-Foldy term so that a charge 
radius of $2.129 \mathrm{fm}$ was obtained. Recoil corrections were taken into account by transforming into the center-of-mass system $[37,38]$. The resulting Coulomb corrections are listed in the $7^{\text {th }}$ column of Table 1 and are in good agreement with the second-order Born result. Finally we have calculated the relative dispersion correction for each scattering energy and scattering angle as discussed before.

3. We then have determined the ratio of deuteron charge form factor to proton charge form factor

$$
R\left(\mathbf{q}^{2}\right) \equiv \frac{G_{E d}}{G_{E p}}=\sqrt{\frac{\sigma_{r e l}}{1+\sum_{i} \Delta_{i}}} / G_{E p}, \quad i=\operatorname{mag}, \text { quad, coul, disp }
$$

using the four-pole fit of $G_{E p}$ given in ref. [35] which describes the $e-p$ cross section measured at Mainz. These data have been confirmed to the $1 \%$-level by independent measurements at Saclay (see Fig. 2 in [36]). The last column in Table 1 lists the values of $R\left(\mathbf{q}^{2}\right)$ together with the associated error (statistical + normalization error).

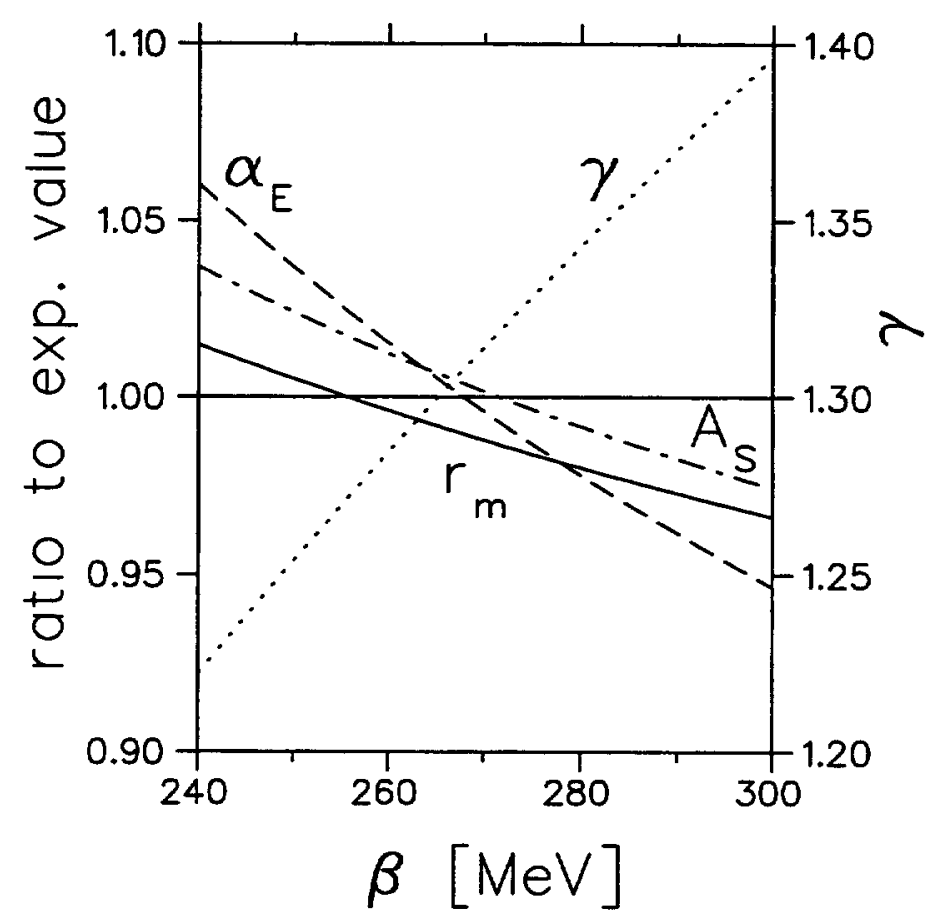

Figure 7: Matter radius $r_{m}$, dipole polarizability $\alpha_{E}$ and asymptotic normalization $A_{S}$ relative to their experimental value $\left(1.967 \mathrm{fm}, 0.6328 \mathrm{fm}^{3}, 0.8845\right.$, respectively) as a function of the Yamaguchi parameter $\beta$ (left hand scale). Also plotted is the exponent $\gamma$ defined in Eq. (45) (right hand scale).

4. We now follow the procedure of Klarsfeld et al. [1]: The ratio $R\left(\mathbf{q}^{2}\right)$ is the body form factor of the deuteron still folded with the neutron charge density and the Darwin-Foldy correction. It thus has the low-q expansion

$$
R\left(\mathbf{q}^{2}\right)=1-\frac{1}{6} \mathbf{q}^{2} r_{x}^{2}+\frac{1}{120} \mathbf{q}^{4} r_{y}^{4}+\ldots
$$


from which we could determine the root-mean-square radius $r_{x}$ by plotting $R\left(\mathbf{q}^{2}\right)$ vs. $\mathbf{q}^{2}$. Klarsfeld $e t$ al. have found that plotting the inverse of $R$ gives rise to a more linear behaviour. This is easily improved by considering $R^{-\gamma}$ and demanding that the $\mathcal{O}\left(\mathbf{q}^{4}\right)$-term vanishes completely. In this way one determines

$$
\gamma=\frac{3}{5} \frac{r_{y}^{4}}{r_{x}^{4}}-1 \simeq \frac{3}{5} \frac{\left\langle r^{4}\right\rangle}{\left\langle r^{2}\right\rangle^{2}}-1
$$

where $\left\langle r^{n}\right\rangle$ are the moments of the matter density. With the Yamaguchi density (A4) one finds $1.25<\gamma<1.35$ in a wide range of $\beta$-values (see Fig. 7) and we adopt $\gamma=1.3$ in our analysis ${ }^{g}$ Thus

$$
r_{x}=\sqrt{\frac{6}{\mathbf{q}^{2}} \frac{R^{-\gamma}-1}{\gamma}}
$$

should be nearly constant as function of $\mathbf{q}^{2}$ and directly gives the radius $r_{x}$. This is indeed the case as can be seen in Fig. 8 .

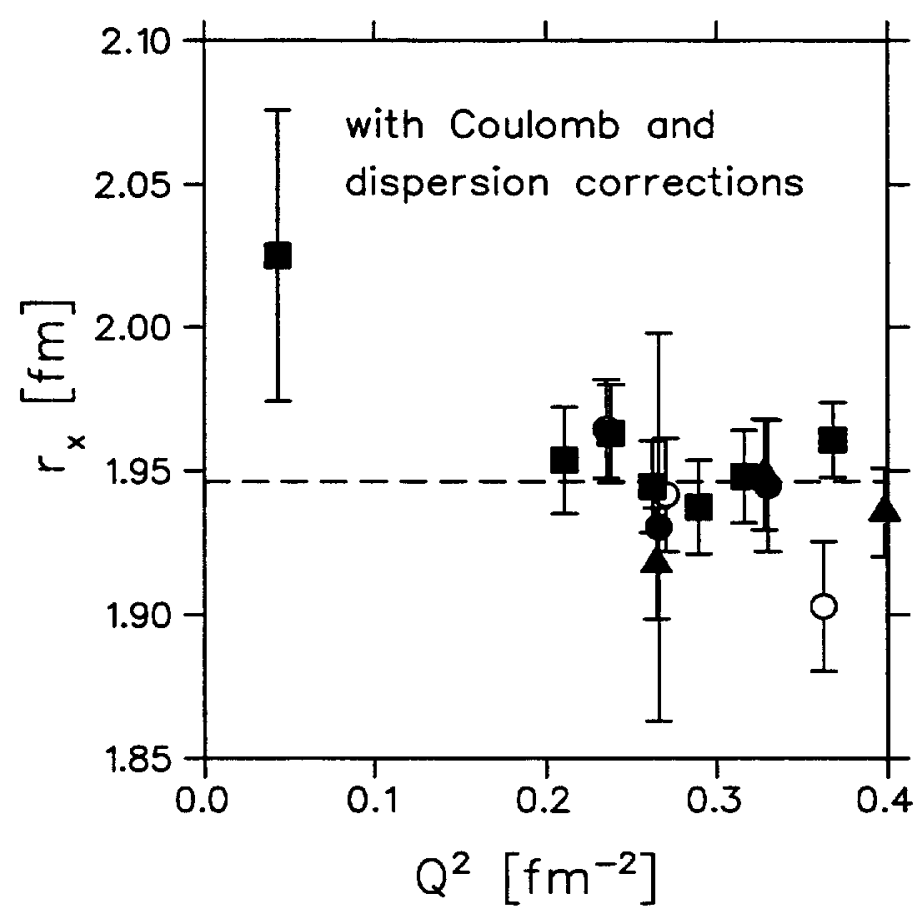

Figure 8: Deuteron radius $r_{x}$ as function of $Q^{2}$ extracted from the 15 experimental $e-d$ cross sections listed in Table 1. The squares, triangles, full dots and open circles denote the 80.1, 149.4, 149.8 and 199.5 MeV data, respectively. The average is indicated by the dashed line.

The weighted average of $r_{x}$ is given in Table 2 for an analysis including Coulomb and dispersion corrections. In all cases a good fit was achieved: the slightly lower $\chi^{2}$ with no corrections is irrelevant.

\footnotetext{
${ }^{g}$ As Donald Sprung has pointed out to us, this fixes the $q^{4}$ coefficient in terms of $r_{x}$ and leads to an underestimate of the error in the radius. However, for the very small momentum transfers which we analyze here, this is not expected to have great influence.
} 


\begin{tabular}{|l|c|c|c|c|}
\hline & $r_{x}[\mathrm{fm}]$ & $\chi^{2} / \mathrm{dof}$ & $r_{E d}[\mathrm{fm}]$ & $r_{c h}[\mathrm{fm}]$ \\
\hline uncorrected & $1.9379(41)$ & 0.68 & $1.9584(41)$ & $2.1209(87)$ \\
with Coulomb corrections & $1.9508(57)$ & 1.00 & $1.9716(56)$ & $2.1327(101)$ \\
$\begin{array}{l}\text { with Coulomb and } \\
\text { dispersion corrections }\end{array}$ & $1.9473(55)$ & 0.93 & $1.9682(54))$ & $2.1296(99)$ \\
\hline
\end{tabular}

Table 2: Deuteron radii extracted from the low-q data from Mainz [33].

However, Sick and Trautmann [17] who analyzed a much greater set of data found a significant improvement of the fit when Coulomb corrections were applied. Also the outcome of the fit is stable if, for example, only the data with $Q^{2}<0.3 \mathrm{fm}^{-2}$ are analyzed: with Coulomb and dispersion corrections one obtains $r_{x}=(1.9506 \pm 0.0079) \mathrm{fm}$ which is fully compatible with the result given in Table 2. We also have listed the radius

$$
r_{E d}^{2}=r_{x}^{2}-r_{n}^{2}-\frac{3}{4 M^{2}}
$$

obtained from Eq. (37) with the value $r_{n}^{2}=(-0.115 \pm 0.004) \mathrm{fm}^{2}$ [39]. Our result (including Coulomb and dispersion corrections)

$$
r_{E d}=1.968(6) \mathrm{fm}
$$

is in very good agreement with a value derived from systematics of the most recent $N N$-potential models and the deuteron asymptotic normalization, $1.967(2) \mathrm{fm}[7]$. If one adds an estimate for the meson-exchange corrections and the small spin-orbit contribution, the theoretical value is raised to 1.971 (2) fm [7] which is still compatible with our result (48). In contrast the value $1.9753(11) \mathrm{fm}$ from atomic laser spectroscopy is much more precise but slightly higher. Finally we add quadratically the proton radius $r_{p}=0.862(12) \mathrm{fm}$ to obtain the charge radius of the deuteron (including Coulomb and dispersion corrections)

$$
r_{c h}=2.130(10) \mathrm{fm} .
$$

Here the increased error is due to the larger uncertainty in the proton charge radius. Table 2 also shows that the inclusion of Coulomb corrections raises the charge radius by $0.012 \mathrm{fm}$ in very good agreement with the result of Sick and Trautmann [17] who analyzed the world data over a much wider range of $Q^{2}$-values and obtained $r_{c h}=2.128(11) \mathrm{fm}$. Our dispersion corrections partially compensate this increase by a reduction of $0.003 \mathrm{fm}$ which is well within the overall uncertainty. 


\section{Summary}

In conclusion, we have evaluated dispersion corrections to elastic electron-deuteron scattering, avoiding for the first time several commonly used drastic approximations. This was achieved by summing explicitly over all excited states of the proton-neutron system in the continuum with the correct weight and using a simple, phenomenological $N N$-potential. We have shown that neither the closure approximation nor the long wavelength approximation are able to reproduce quantitatively the exact results obtained by numerical integration. Contrary to naive expectations based on the fact that the deuteron is a loosely bound and easily polarizable nuclear system, our dispersion corrections turn out to be small - much less in magnitude than given by the few previous estimates in the literature. In addition, the corrections are definitely negative, i.e. the non-static cross section is smaller than the one calculated without dispersion corrections. This means that the true rms-radius is smaller than the one obtained by analyzing the scattering data in a static framework. This was born out quantitatively by analyzing the experimental cross sections by Simon et al. at low momentum transfer where our results are deemed to be most reliable. We found that the decrease in the matter (or charge) radius of the deuteron due to the inclusion of dispersion corrections is about $2-3$ times smaller than the present experimental error and that it does not remove the slight disagreement between the values derived from electron scattering and laser spectroscopy.

Although we believe that our calculation of dispersion corrections is a substantial step forward in controlling these corrections, it could be improved in several respects:

a) Inclusion of transverse virtual excitations within our simple S-wave model of the deuteron.

b) Inclusion of the $\mathrm{D}$-wave component, i.e. the tensor force in a separable potential [40].

c) Calculation with a realistic potential.

As the deuteron dispersion corrections are rather small and in view of the complexity of the numerical calculation (which roughly is one to two orders of magnitude larger than a calculation of the nuclear polarization shift) it is probably the best strategy to concentrate on point a), i.e. to include transverse excitations within the simplified $\mathrm{S}$-wave model. This seems a fairly straightforward extension of our present calculations and is left for the future.

Acknowledgement: We thank Joan Martorell for very helpful comments and suggestions which were instrumental to the analysis of the electron scattering data and for information about the theoretical status of the deuteron radius. Donald Sprung raised pertinent questions and carefully read a first draft of this paper for which we are very grateful. We are also indebted to Valeri Markushin for a critical reading of the manuscript. 


\section{Appendix}

\section{A Generalized structure function for the Yamaguchi potential}

The Schrödinger equation can be solved analytically for the separable Yamaguchi potential, giving the ground state wave function of the deuteron

$$
\psi_{0}(p)=\frac{N}{\left(\alpha^{2}+p^{2}\right)\left(\beta^{2}+p^{2}\right)} .
$$

Here $\alpha=\sqrt{M \omega_{0}}=45.701 \mathrm{MeV}$ and $p=|\mathbf{p}|$. The normalization $\int d^{3} p\left|\psi_{0}(p)\right|^{2}=1$ requires

$$
N^{2}=\frac{\alpha \beta}{\pi^{2}}(\alpha+\beta)^{3} \text {. }
$$

The wave function in $r$-space has the Hulthén form

$$
\psi_{0}(r)=\sqrt{\frac{\pi}{2}} \frac{N}{\beta^{2}-\alpha^{2}} \frac{1}{r}\left(e^{-\alpha r}-e^{-\beta r}\right)
$$

and the ground state density is given by

$$
\rho(r)=8\left|\psi_{0}(2 r)\right|^{2}=\frac{\pi N^{2}}{\left(\beta^{2}-\alpha^{2}\right)^{2}} \frac{1}{r^{2}}\left(e^{-2 \alpha r}-e^{-2 \beta r}\right)^{2} .
$$

The elastic (body) form factor is easily found to be

$$
F_{0}(q)=\frac{4 \pi^{2} N^{2}}{\left(\beta^{2}-\alpha^{2}\right)^{2}} \frac{1}{q} \arctan \left\{\frac{4(\alpha+\beta)(\beta-\alpha)^{2} q}{\left[4 \alpha(\alpha+\beta)+q^{2} / 2\right]\left[4 \beta(\alpha+\beta)+q^{2} / 2\right]+(\beta-\alpha)^{2} q^{2}}\right\} .
$$

For convenience we list the analytical expressions for the moments of the density (A4)

$$
\left\langle r^{n}\right\rangle=n ! \frac{\alpha \beta}{2^{2 n}} \frac{(\alpha+\beta)^{3}}{\left(\beta^{2}-\alpha^{2}\right)^{2}}\left[\frac{1}{\alpha^{n+1}}+\frac{1}{\beta^{n+1}}-\frac{2^{n+2}}{(\alpha+\beta)^{n+1}}\right]
$$

the asymptotic normalization

$$
A_{S}=\sqrt{2 \alpha \beta} \frac{(\alpha+\beta)^{3 / 2}}{\beta^{2}-\alpha^{2}}
$$

and the electric dipole polarizability [26]

$$
\alpha_{E}=\frac{e^{2}}{96} \frac{M}{\alpha^{4} \beta^{2}(\alpha+\beta)^{3}}\left[3 \beta^{5}+18 \beta^{4} \alpha+51 \beta^{3} \alpha^{2}+96 \beta^{2} \alpha^{3}+48 \beta \alpha^{4}+8 \alpha^{5}\right] .
$$

The inelastic part of the generalized longitudinal structure function is obtained from the analytic solution of the full Green function as ${ }^{h}$

$$
S_{L}{ }^{\text {inelastic }}\left(\mathbf{q}_{1}, \mathbf{q}_{2}, \omega\right)=S_{\text {free }}-\frac{1}{\pi} \operatorname{Im}\left\{\lambda M \frac{1}{1+\lambda C\left(\omega+\omega_{0}\right)} D\left(\omega+\omega_{0}, q_{1}\right) D\left(\omega+\omega_{0}, q_{1}\right)\right\}
$$

\footnotetext{
${ }^{h}$ For details see Ref. [19].
} 
with

$$
\begin{aligned}
C(k) & =\frac{\pi^{2}}{\beta(k+i \beta)^{2}} \\
D(k, q) & =\frac{\pi N}{\alpha^{2}-\beta^{2}} \frac{1}{q}\{I(k, q, \alpha, \beta)-I(k,-q, \alpha, \beta)+I(k,-q, \beta, \beta)-I(k, q, \beta, \beta)\} .
\end{aligned}
$$

Here $k^{2}=M\left(\omega+\omega_{0}\right), \lambda=\beta(\beta+\alpha)^{2} / \pi^{2}$ and

$$
I(k, q, \alpha, \beta)=\frac{\pi i}{k^{2}+\beta^{2}} \ln \left[\frac{\alpha^{2}+\left(k+\frac{q}{2}\right)^{2}}{\alpha^{2}+\left(i \beta+\frac{q}{2}\right)^{2}} \frac{\beta^{2}+\left(i \alpha-\frac{q}{2}\right)^{2}}{-k^{2}+\left(i \alpha-\frac{q}{2}\right)^{2}}\right] .
$$

By means of partial fractions the non-interacting part

$$
S_{\text {free }}=\int d^{3} p \psi_{0}\left(\mathbf{p}-\frac{\mathbf{q}_{2}}{2}\right) \psi_{0}\left(\mathbf{p}-\frac{\mathbf{q}_{1}}{2}\right) \delta\left(\omega+\omega_{0}-\frac{p^{2}}{M}\right)
$$

can be reduced to

$$
S_{\text {free }}=M N^{2} \frac{1}{\left(\beta^{2}-\alpha^{2}\right)^{2}}[J(\alpha, \alpha)-J(\alpha, \beta)-J(\beta, \alpha)+J(\beta, \beta)]
$$

with

$$
J(a, b)=\int d^{3} p \frac{1}{a^{2}+\left(\mathbf{p}-\mathbf{q}_{1} / 2\right)^{2}} \frac{1}{b^{2}+\left(\mathbf{p}-\mathbf{q}_{2} / 2\right)^{2}} \delta\left(k^{2}-p^{2}\right) .
$$

Combining the denominators with the Feynman procedure, the integral $J(a, b)$ can be finally brought into the form

$$
J(a, b)=2 \pi k \int_{0}^{1} d x \frac{1}{A x^{2}+B x+C},
$$

which has standard analytical solutions in terms of elementary functions depending on the values of the parameters

$$
\begin{aligned}
& A=\left(a^{2}-b^{2}+\frac{q_{1}^{2}}{4}-\frac{q_{2}^{2}}{4}\right)^{2}-k^{2}\left(\mathbf{q}_{1}-\mathbf{q}_{2}\right)^{2} \\
& B=2\left(a^{2}-b^{2}+\frac{q_{1}^{2}}{4}-\frac{q_{2}^{2}}{4}\right)\left(b^{2}+\frac{q_{2}^{2}}{4}+k^{2}\right)-2 k^{2} \mathbf{q}_{2} \cdot\left(\mathbf{q}_{1}-\mathbf{q}_{2}\right) \\
& C=\left(b^{2}+\frac{q_{2}^{2}}{4}+k^{2}\right)^{2}-k^{2} q_{2}^{2} .
\end{aligned}
$$




\section{References}

[1] S. Klarsfeld, J. Martorell, J. A. Oteo, N. Nishimura and D. W. L. Sprung, Nucl. Phys. A 456 (1986) 373.

[2] D. W. L. Sprung, Hua Wu and J. Martorell, Phys. Rev. C 42, (1990) 863.

[3] R. K. Bhaduri, W. Leidemann, G. Orlandini and E. L. Tomusiak, Phys. Rev. C 42 (1990) 1867.

[4] D. W. L. Sprung and Hua Wu, Acta Phys. Polon. B 24 (1993) 503.

[5] C. W. Wong, Int. J. Mod. Phys. E 3 (1994) 821.

[6] A. J. Buchmann, H. Henning and P. U. Sauer, Few-Body Sys. 21 (1996) 149.

[7] J. L. Friar, J. Martorell and D. W. L. Sprung, nucl-th/9707016 (July 1997); Phys. Rev. A 56 (1997) December issue.

[8] F. Schmidt-Kaler, D. Leibfried, M. Weitz and T. W. Hänsch, Phys. Rev. Lett. 70 (1993) 2261.

[9] K. Pachucki et al., J. Phys. B 29 (1996) 177.

[10] T. W. Hänsch, invited talk at the $12^{\text {th }}$ Interdisciplinary Laser Science Conference, Rochester, N. Y., Oct. 20, 1996.

[11] K. Pachucki, M. Weitz and T. W. Hänsch, Phys. Rev. A 49 (1994) 2255.

[12] Y. Lu and R. Rosenfelder, Phys. Lett. B 319 (1993) 7; B 333 (1994) 564 (E).

[13] W. Leidemann and R. Rosenfelder, Phys. Rev. C 51 (1995) 427.

[14] J. Martorell, D. W. L. Sprung and D. C. Zheng, Phys. Rev. C 51 (1995) 1127.

[15] J. L. Friar and G. L. Payne, Phys. Rev. C 56 (1997) 619.

[16] E. Zavattini et al., PSI proposal R-93-06.1 (1993).

[17] I. Sick and D. Trautmann, Phys. Lett. B 375 (1996) 16.

[18] J. L. Friar and M. Rosen, Ann. Phys. (N.Y.) 87 (1974) 289.

[19] T. Herrmann: Dispersionskorrekturen in der elastischen Elektron-Deuteron-Streuung, diploma thesis (in German), Universität Kaiserslautern (May 1997).

[20] A. Bottino and G. Ciochetti, Phys. Lett. 34 B (1971) 187; Nucl. Phys. A 178 (1972) 593.

[21] R. R. Lewis, Phys. Rev. 102 (1956) 537.

[22] S. D. Drell and R. H. Pratt, Phys. Rev. 125 (1962) 1394.

[23] R. H. Dalitz,, Proc. Roy. Soc. A 206 (1951) 509.

[24] Y. Yamaguchi, Phys. Rev. 95 (1954) 1628. 
[25] J. L. Friar and G. L. Payne, Phys. Rev. C 55 (1997) 2764.

[26] J. L. Friar and S. Fallieros, Phys. Rev. C 29 (1984) 232.

[27] V. Z. Yankus, Phys. Rev. 102 (1956) 1586.

[28] N. A. Krall and E. E. Salpeter, Phys. Rev. 115 (1959) 457.

[29] M. Gourdin, Nuov. Cim. 28 (1963) 533.

[30] R. J. Adler, Phys. Rev. 141 (1966) 1499.

[31] F. Gross, Phys. Rev. 142 (1966) 1025; 152 (1966) 1517 (E).

[32] T. deForest and J. D. Walecka, Adv. Phys. 15 (1966) 1.

[33] G. G. Simon, Ch. Schmitt and V. H. Walther, Nucl. Phys. A 364 (1981) 285.

[34] R. W. Berard et al. Phys. Lett. 47 B (1973) 355.

[35] G. G. Simon, Ch. Schmitt, F. Borkowski and V. H. Walther, Nucl. Phys. A 333 (1980) 381.

[36] S. Platchkov et al., Nucl. Phys. A 510 (1990) 740.

[37] L. L. Foldy, K. W. Ford and D. R. Yennie, Phys. Rev. 113 (1959) 1147.

[38] J. L. Friar, Ann. Phys. (N.Y.) 98 (1976) 490.

[39] S. Kopecky et al., Phys. Rev.C 56 (1997) 2229.

[40] Y. Yamaguchi and Y. Yamaguchi, Phys. Rev. 95 (1954) 1635. 
